\title{
Exact solution for unsteady free convection flow of Casson fluid in vertical channel
}

\author{
Ahmad Qushairi Mohamad ${ }^{1}$, Lim Yeou Jiann ${ }^{1}$, Sharidan Shafie ${ }^{1, *}$, Ilyas Khan ${ }^{2}$, and \\ Zulkhibri Ismail ${ }^{3}$ \\ ${ }^{1}$ Department of Mathematical Sciences, Faculty of Science, Universiti Teknologi Malaysia, 81310 \\ UTM Johor Bahru, Johor, Malaysia \\ ${ }^{2}$ Faculty of Mathematics and Statistics, Ton Duc Thang University, Ho Chi Minh City, Vietnam. \\ ${ }^{3}$ Faculty of Industrial Sciences and Technology, Universiti Malaysia Pahang, 26300 Kuantan, Pahang, \\ Malaysia
}

\begin{abstract}
The influence of free convection flow on unsteady Casson fluid over a vertical channel is studied in this paper. The governing equation consists of partial differential momentum and energy equations have been transformed into non-dimensional equations by using the appropriate nondimensional variables. The expression of exact solutions for nondimensional velocity and temperature profiles are obtained by using the Laplace transform method. The obtained exact solutions are found satisfied all the initial and boundary conditions. The behaviour of velocity and temperature profiles influenced by Casson fluid parameter, Grashof number, Prandtl number and time are plotted graphically and presented in tables. It can be observed that, the velocity profile and boundary layer thickness decrease when the values of Casson fluid parameter and Prandtl number increase. This is due to the increase of viscous force and retard the movement of the velocity. The present result is validated when the obtained exact solution of velocity is compared with published result and found in a good agreement.
\end{abstract}

\section{Introduction}

Heat transfer analysis in vertical channel (two parallel vertical plates) has been studied by a few researchers due to their applications. For example cooling of electronic equipment, solar collectors, passive heating of buildings and channel nuclear reactors. One of the earliest researchers that investigated the fully developed of free convection heat transfer in vertical channel is Ostrach [1,2]. He focused on the steady free and forced convective laminar flows in constant wall temperature and solved it by using the Fourier series. Then, in early year of 2000, most of the researchers that dealing with channel problem are using the Laplace transform method, in order to obtain the exact solutions of the problem studied. Paul et al. [3] obtained the exact solutions for free convection flow of incompressible viscous fluid in vertical channel with constant heat flux effect whereas Basant [4] solved the problem of free convection flow on magnetic effect in electrically conducting viscous

\footnotetext{
* Corresponding author: sharidan@utm.my
} 
fluid through a vertical channel. Then, Narahari [5] extended the problem of Paul et al. [4] by considering the effect of radiation. Narahari [6] produced the new problem by adding mass transfer on fluid flow problem without the effect of constant heat flux and radiation. With the great knowledge of exact solution in vertical channel, once again, Narahari and Vijay [7] published the problem of free convection flow of viscous fluid with the effect of ramped wall temperature. They found that, the velocity and temperature profiles in the case of ramped temperature plate is always less than that of the case of an isothermal plate. Then, Jha $[8,9]$ studied the free convection flow of viscous fluid in heat and mass transfer with the dufour effect. Finally, the problems on rotating viscous fluid in vertical channel are also studied by Seth et al. [10] (porous channel and magnetic effect), Seth et al. [11] (porous channel an, magnetic and Hall current effects) and Seth et al. [12] (porous medium, magnetic and heat transfer effects).

From the above discussion, all researchers are focused on Newtonian fluid or viscous fluid. Therefore, in this study we would like to concentrate on non-Newtonian fluid, especially the problem on Casson fluid. This fluid was introduced by Casson in 1959 to study the prediction of the flow behaviour of pigment-oil suspensions [13]. Then, Bhattacharyya et al. [14] said that, Casson fluid consist of small shear stress and can be marked as a purely viscous fluid with high viscosity. Besides that, this Casson fluid also modelled by [15] for the pathological situation of blood flow problem. Due to that application, Asma et al. [16] observed the new problem on unsteady free convection flow of Casson fluid in vertical constant wall temperature. Then, they extended their problem by considering the effects of magnetohydrodynamics (MHD) and porous medium [17]. Recently, Mohamad et al. [18] continue the idea of [17] by adding the effect of radiation, mass transfer and arbitrary wall shear stress. Motivated by the above studies, the present work focused on unsteady free convection flow of Casson fluid in infinite vertical channel. The exact solutions are obtained by using the Laplace transform method. The behaviour of important physical parameters are plotted graphically and discussed in details. The present work is validated by comparing with published result [6].

\section{Problem formulation}

Consider the unsteady free convection flow of incompressible Casson fluid in two infinite vertical plates separated by a distance $d$ or vertical channel with constant temperature. The $x^{\prime}$-axis is taken along one of the plates in the vertically upward direction and the $y^{\prime}$-axis is taken normal to the plates. Initially, at time $t^{\prime} \leq 0$, the two plates and the fluid are assumed to be at the same temperature $T_{d^{\prime}}$. Then, at time $t^{\prime}>0$, the temperature of the plate at $y^{\prime}=0$ are raised to $T_{w}{ }^{\prime}$ whereas the plate at $y^{\prime}=d$ is stationary and maintained at a constant temperature $T_{d}{ }^{\prime}$. Under the usual Boussinesq's approximation, the corresponding partial differential equations for momentum and energy are given as

$$
\begin{array}{r}
\rho \frac{\partial u^{\prime}}{\partial t^{\prime}}=\mu\left(1+\frac{1}{\gamma}\right) \frac{\partial^{2} u^{\prime}}{\partial y^{\prime 2}}+\rho g_{x} \beta\left(T^{\prime}-T_{d}^{\prime}\right), \\
\rho C_{p} \frac{\partial T^{\prime}}{\partial t^{\prime}}=k \frac{\partial^{2} T^{\prime}}{\partial y^{\prime 2}},
\end{array}
$$

together with the initial and boundary conditions,

$$
\begin{array}{rrr}
u^{\prime}\left(y^{\prime}, 0\right)=0, & T^{\prime}\left(y^{\prime}, 0\right)=T_{d} ; & 0 \leq y^{\prime} \leq d, \\
u^{\prime}\left(0, t^{\prime}\right)=0, & T^{\prime}\left(0, t^{\prime}\right)=T_{w} ; & t^{\prime}>0, \\
u^{\prime}\left(d, t^{\prime}\right)=0, T^{\prime}\left(d, t^{\prime}\right)=T_{d} ; & t^{\prime}>0,
\end{array}
$$


where $u^{\prime}$ is the velocity component along $x$-axes, $\rho$ is the density of fluid, $\mu$ is the dynamic viscosity of fluid, $\gamma$ is the non-Newtonian Casson parameter, $g_{x}$ is the gravitational acceleration, $\beta$ is the heat transfer coefficient, $T^{\prime}$ is the temperature, $C_{p}$ is the specific heat of the fluid at constant pressure and $k$ is the thermal conductivity of the fluid. In order to simplify equations (1)-(3), introduce the non-dimensional variables

$$
u=\frac{u^{\prime} d}{v}, \quad y=\frac{y^{\prime}}{d}, \quad t=\frac{t^{\prime} v}{d^{2}}, \quad T=\frac{T^{\prime}-T_{d}^{\prime}}{T_{w}^{\prime}-T_{d}^{\prime}},
$$

and substitute (4) into equations (1)-(3), obtained the non-dimensional partial differential equations as

$$
\begin{aligned}
& \frac{\partial^{2} u}{\partial y^{2}}-a \frac{\partial u}{\partial t}=-a G r T \\
& \frac{\partial^{2} T}{\partial y^{2}}-\operatorname{Pr} \frac{\partial T}{\partial t}=0 \\
& u(y, 0)=0, \quad T(y, 0)=0 ; \quad 0 \leq y \leq 1, \\
& u(0, t)=0, \quad T(0, t)=1 ; \quad t>0, \\
& u(1, t)=0, T(1, t)=0 ; \quad t>0,
\end{aligned}
$$

where ${ }_{a=\frac{\gamma}{1+\gamma}}$ is the constant parameter, $G r=\frac{g_{x} \beta d^{3}}{v^{2}}\left(T_{w}^{\prime}-T_{d}^{\prime}\right)$ is the Grashof number and $\operatorname{Pr}=\frac{\mu C_{p}}{k}$ is the Prandtl number.

\section{Problem solution}

In order to obtain the exact solution of equations (5) and (6), apply Laplace transform into equations (5) and (6) subjected to the initial equations (7), yields

$$
\begin{aligned}
& \frac{d^{2} \bar{u}}{d y^{2}}-a q \bar{u}=-a G r \bar{T}, \\
& \frac{d^{2} \bar{T}}{d y^{2}}-\operatorname{Pr} q \bar{T}=0,
\end{aligned}
$$

where $\bar{u}(y, q)$ is the Laplace velocity, $\bar{T}(y, q)$ is the Laplace temperature and $q$ is the transformation variable. The corresponding Laplace transform for boundary conditions are

$$
\begin{gathered}
\bar{u}(0, q)=0, \quad \bar{T}(0, q)=\frac{1}{q}, \\
\bar{u}(1, q)=0, \quad \bar{T}(1, q)=0 .
\end{gathered}
$$
give:

The inverse Laplace transform of equations (8) and (9) by imposed of equations (10),

$$
\begin{gathered}
u(y, t)=b_{1} \sum_{n=0}^{\infty}\left[u_{1}(y, t)-u_{2}(y, t)\right]-b_{1} \sum_{n=0}^{\infty}\left[u_{3}(y, t)-u_{4}(y, t)\right] \\
T(y, t)=\sum_{n=0}^{\infty}\left[\operatorname{Erfc}\left(\frac{a_{3} \sqrt{\operatorname{Pr}}}{2 \sqrt{t}}\right)-\operatorname{Erfc}\left(\frac{a_{4} \sqrt{\operatorname{Pr}}}{2 \sqrt{t}}\right)\right]
\end{gathered}
$$


where

$$
\begin{gathered}
u_{1}(y, t)=\left(\frac{a_{3}^{2}}{2}+t\right) \operatorname{Erfc}\left(\frac{a_{3}}{2 \sqrt{t}}\right)-a_{3} \sqrt{\frac{t}{\pi}} \exp \left(-\frac{a_{3}^{2}}{4 t}\right), \\
u_{2}(y, t)=\left(\frac{a_{4}^{2}}{2}+t\right) \operatorname{Erfc}\left(\frac{a_{4}}{2 \sqrt{t}}\right)-a_{4} \sqrt{\frac{t}{\pi}} \exp \left(-\frac{a_{4}^{2}}{4 t}\right), \\
u_{3}(y, t)=\left(\frac{a_{5}^{2}}{2}+t\right) \operatorname{Erfc}\left(\frac{a_{5}}{2 \sqrt{t}}\right)-a_{5} \sqrt{\frac{t}{\pi}} \exp \left(-\frac{a_{5}^{2}}{4 t}\right), \\
u_{4}(y, t)=\left(\frac{a_{6}^{2}}{2}+t\right) \operatorname{Erfc}\left(\frac{a_{6}}{2 \sqrt{t}}\right)-a_{6} \sqrt{\frac{t}{\pi}} \exp \left(-\frac{a_{6}^{2}}{4 t}\right), \\
a_{3}=(2 n+y) \sqrt{a}, a_{4}=(2 n+2-y) \sqrt{a}, \quad a_{5}=(2 n+y) \sqrt{\operatorname{Pr}}, \quad a_{6}=(2 n+2-y) \sqrt{\operatorname{Pr}} \text { and } b_{1}=\frac{a G r}{\operatorname{Pr}-a} .
\end{gathered}
$$

\section{Results and discussion}

In order to check the accuracy of the present results (11), the limiting case of the present result is compared with published results by Narahari [6]. This comparison is shown in figure 1. It is found that our limiting result (11) is identical to (10) obtained by [6]. This confirms the accuracy of our obtained results. To understand the behaviour of velocity and temperature profiles, the numerical results of these profiles are computed and plotted for various parameters of interest such as Casson parameter $\gamma$, Grashof number Gr, Prandtl number Pr, and time parameter $t$. The effect of $\gamma$ on velocity profiles are plotted in figure 2 . From the observation, the velocity decreases when the values of $\gamma$ increases. This is because, the viscosity of the fluid will be higher when the value of $\gamma$ increases. Figure 3 shows that the velocity is increasing when the values of $G r$ increases. From the definition, the $G r$ is ratio of buoyancy force to viscous force. Therefore, increase in $G r$ will increase the buoyancy force and enhance the velocity of fluid to increase. The similar behaviour of Pr can be observed in velocity (Figure 4) and temperature (Figure 6) profiles. It is shown that, increase in $\operatorname{Pr}$ will decrease both profiles. This is due to increase in viscous force. Lastly, the behaviour of $t$ is plotted in figures 5 and 7 where the velocity and temperature profiles increase when the $t$ increases.

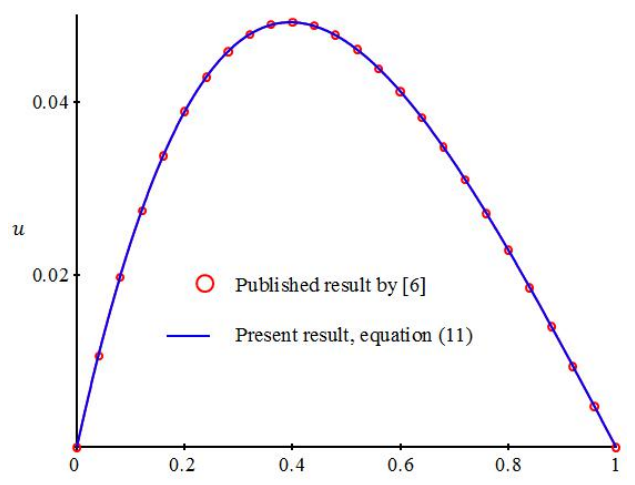

$y$

Fig. 1. Comparison of the present results (see equation (11)) when $\gamma \rightarrow \infty$ with those obtained by Narahari [6] when $S c=G m=0$. 

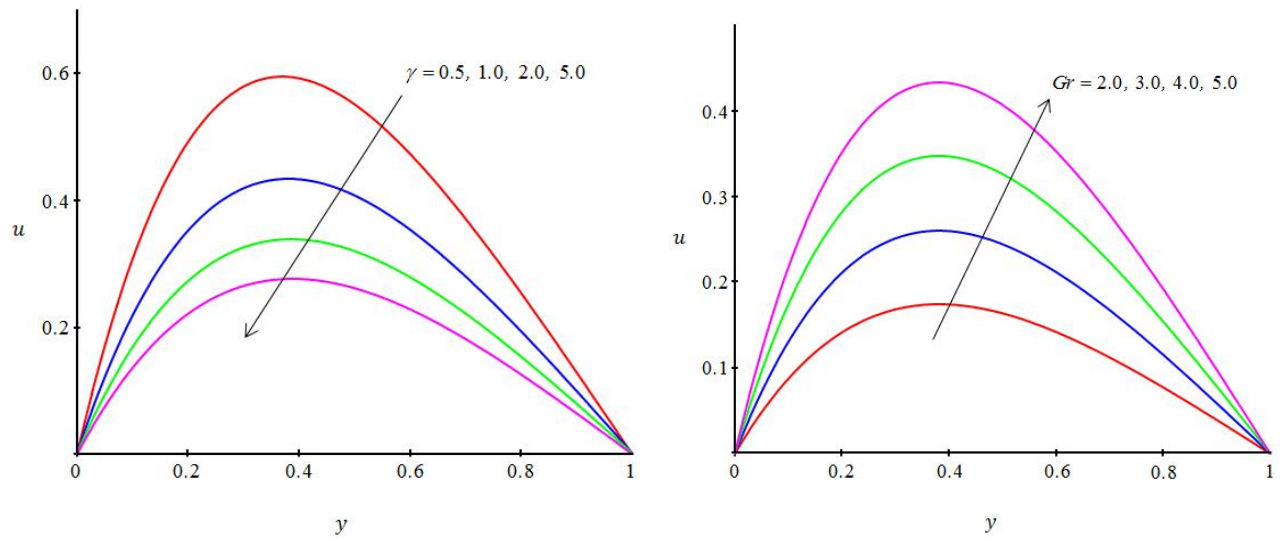

Fig. 2. Velocity profiles for different values of $\gamma$.

Fig. 3. Velocity profiles for different values of $G r$.
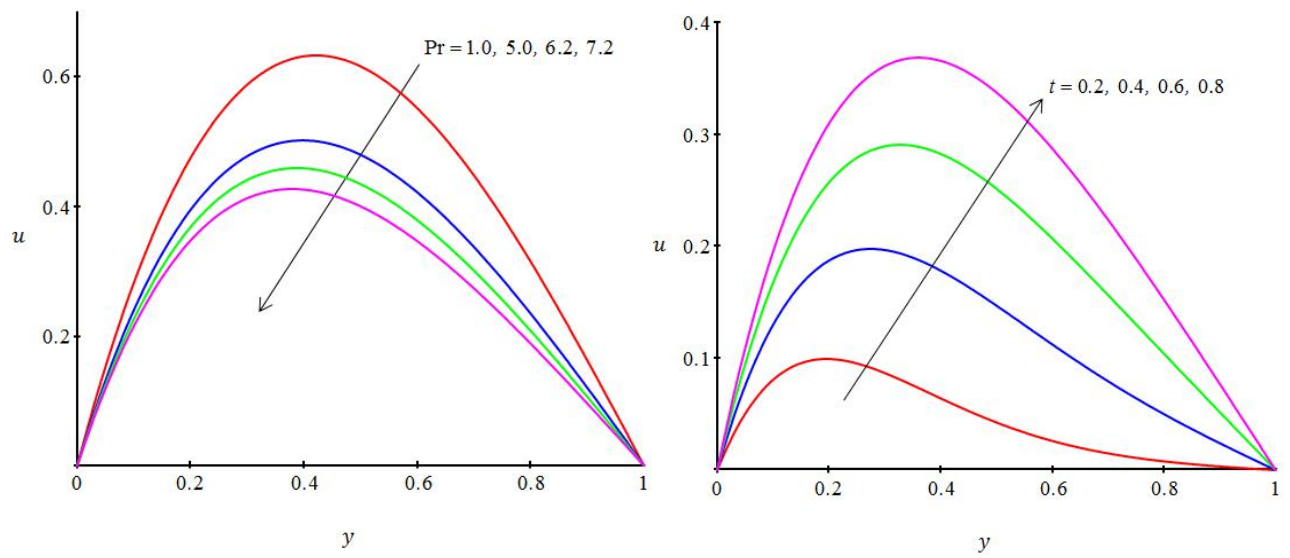

Fig. 4. Velocity profiles for different values of Pr.

Fig. 5. Velocity profiles for different values of $t$.
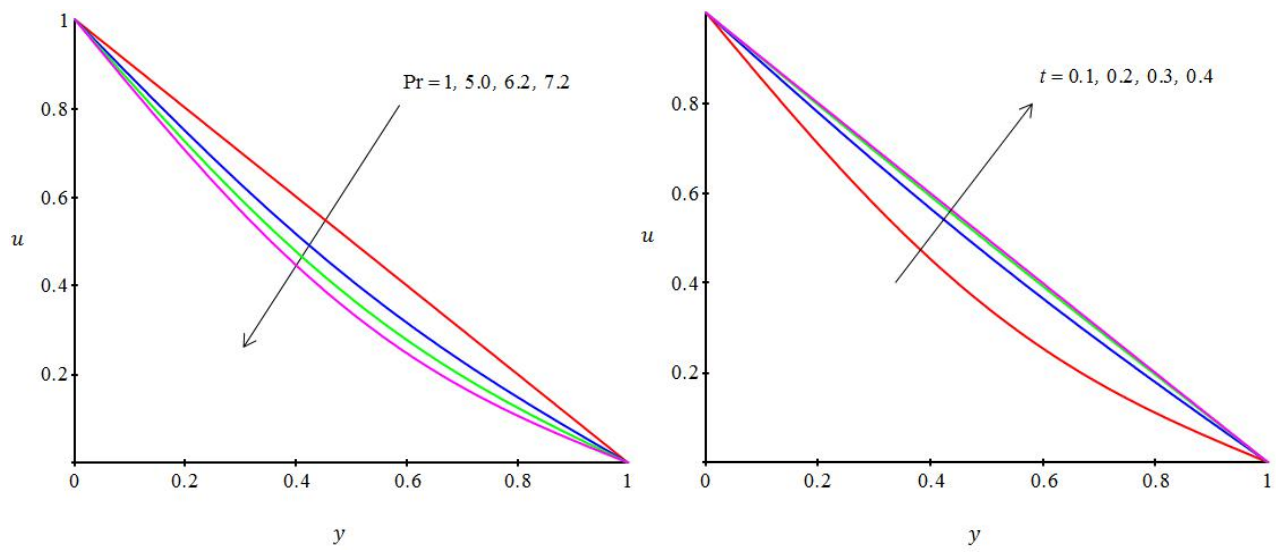

Fig. 6. Temperature profiles for different values of $\mathrm{Pr}$ values of $t$.

Fig. 7. Temperature profiles for different

\section{Conclusion}


The problem on unsteady free convection flow of Casson fluid in vertical channel has been investigated. The exact solutions have been obtained by using the Laplace transform method. The results for velocity and temperature profiles are plotted graphically with the effects of Casson parameter $\gamma$, Grashof number $G r$, Prandtl number Pr, time parameter $t$ and analyse in details. The velocity and temperature increase when $G r$ and $t$ increase but decrease when $\gamma$ and $\operatorname{Pr}$ increase. Solution (11) is found in excellent agreement with those obtained by Narahari [6].

\section{Acknowledgement}

The authors would like to acknowledge Ministry of Higher Education (MOHE) and Research Management Centre-UTM for the financial support through vote numbers 4F713, $13 \mathrm{H} 74$ and $15 \mathrm{H} 80$ for this research.

\section{References}

1. Ostrach S 1952. NASA Report 2863

2. Ostrach S 1954. NASA Report 3141

3. Paul T, Jha BK and Singh AK 1996. Heat Mass Transf 3261

4. Basant KJ 1998. Indian J Pure Appl Mathb29 441

5. Narahari M 2009. Proc $11^{\text {th }}$ WSEAS 73

6. Narahari M 2008. Proc WCE 2

7. Narahari M and Vijay RR 2009. Proc ASME 1

8. Jha BK and Ajibade AO 2010. Proc IMechE 22491

9. Jha Bk 2011. J. Heat Transfer 1331

10. Seth GS, Ansari MS and Nandkeolyar R 2011. J Appl Fluid Mechanics 495

11. Seth GS, Singh JK and Mahato GK 2012. Int J Appl Mechanics 41

12. Seth GS, Kumbhakar B and Sharma R 2015. Int J Appl Mechanics 8767

13. Casson N 1959. A flow equation for the pigment oil suspensions of the pritning ink type, in: Rheology of Disperse Systems vol. 1959( Pergamon, New York) pp84-102

14. Bhattacharyya K, Hayat $\mathrm{T}$ and Alsaedi A 2013. Z Angew Math Mech 101

15. Boyd J, Buick JM and Green S 2007. Phys Fluids 19

16. Asma K, Ilyas K and Sharidan S 2015. Mal J Fund Appl Sci 1128

17. Asma K, Ilyas K, Arshad K and Sharidan S 2015. Eng Sci Technol 18309

18. Mohamad AQ, Ilyas K, Jiann LY, Arshad K, Ilias MR and Sharidan S 2017. J. Nanofluids 61 